\title{
Intelligent Chatbot for Guided Navigation of Repository Contend
}

\author{
Miss. Anjali Mishra ${ }^{1}$, Miss. Shruti Sapre ${ }^{2}$, Miss. Shruti Shinde ${ }^{3}$, \\ Miss. Shreya Nahar ${ }^{4}$, Prof. S.N Shelke ${ }^{5}$ \\ Comp Dept, Stes, Sinhgad Academy of Engineering, Pune, India ${ }^{1-4}$ \\ Professor, Comp Dept, Stes, Sinhgad Academy of Engineering, Pune, India
}

\begin{abstract}
Chatbot's are computer application that interact with users using normal language. This technology started in the 1960 and the aim of technology was to see if chatbot systems could fool users that they were real humans. However, chatbot systems are not only built to copy human conversation, and entertain users. In this paper, we examine other applications where chatbots could be useful such as education, information retrival, business, and e-commerceA chatbot aims to make a conversation between human and machine. The machine has been embedded knowledge to determine the sentences and making a decision itself as response to answer a question. The response purpose is matching the input sentence from user. From input sentence, it will be scored to get the similarity of sentences, the higher score obtained the more similar of reference sentences. The sentence similarity calculation in this paper using web application which divides input sentence as two letters of input sentence. The information of chatbot are stored in the database. The chatbot consists of core and interface. The database has been considered as information storage and interpreter has been considered as stored programs of function and procedure sets for pattern-matching requirement. The interface is standalone which has been built using programming language of Java. In our system Natural Language Processing is used. Our system understands content of conversation based on recent Natural Language Processing (NLP).
\end{abstract}

Keywords: Chatbot, User, Communication, MySQL, Entertain

\section{INTRODUCTION}

The need of conversational agents has become important with the widespread use of personal machines with the wish to communicate and the desire of their makers to provide natural language interfaces. In the computer age, there are a vast quantity of documents rapidly created to meet user's needs. There are large amount of digital documents on the Internet, which are used in various different ways. All the documents containing the information support many type of decision-making. However, many questions are still unanswered, which brings the idea of constructing a system for questioning and answering doubts. This clearly brings us to see that the obstacle of building such system which is a knowledge provider.

Nevertheless, in several condition e.g. in vacation or during a rest, the seller may be unable to communicate with the customer. But for a user, it may take time to answer customer's doubts. As the number of consumers increase the waiting time increases as well, which results in poor customer satisfaction. As a solution, the seller can employ several customer services to answer any questions from customer. However, it involves additional costs for paying customer service officers. As an alternative and better solution, we can develop a system that can receive and understand the question from customer and automatically, give the best response with respect to that question.

NLP provide set of human language technology tool it support the number of major human language. In Chat bot NLP provides Foundational Building Block for higher level and domain specific text under sting Application.

Automated live chat support service is the solution for such problem. Chatbot will take care of user questions and problems. It will give the solution for the same. A chatbot is well designed to answer in a such a way both general questions and FAQs about a particular domain.

\section{LITERATURE SURVEY}

"Chatbots and conversational agents: A bibliometric analysis", H. N. Io ;C. B. Lee, : 2017 IEEE International Conference on Industrial Engineering and Engineering Management (IEEM)

Chatbots are replacing some of the jobs that are traditionally performed by human workers, such as online customer service agents and educators. From the initial stage of rule-based chatbots to the era of rapid development in artificial intelligence (AI), the performance of chatbots keeps improving. Chatbots can nowadays "chat" like a human 
Vol. 8, Issue 5, May 2019

being and they can learn from experience. The purpose of this research is to examine the past research on chatbots (also known as conversational agents) using the quantitative bibliometric analysis. The contribution of this research is to help researchers to identify research gaps for the future research agenda in chatbots. The results of the analysis found a potential research opportunity in chatbots due to the emergence of the deep learning technology. This new technology may change the direction of future research in chatbots. Several recommendations for future research are provided based on the results obtained from our analysis.

\section{“An intelligent web-based voice chat bot", S. J. du Preez ; M. Lall ; S. Sinha}

This paper presents the design and development of an intelligent voice recognition chat bot. The paper presents a technology demonstrator to verify a proposed framework required to support such a bot (a Web service). While a black box approach is used, by controlling the communication structure, to and from the Web-service, the Web-service allows all types of clients to communicate to the server from any platform. The service provided is accessible through a generated interface which allows for seamless XML processing; whereby the extensibility improves the lifespan of such a service. By introducing an artificial brain, the Web-based bot generates customized user responses, aligned to the desired character. Questions asked to the bot, which is not understood is further processed using a third-party expert system (an online intelligent research assistant), and the response is archived, improving the artificial brain capabilities for future generation of responses.

"The chatbot feels you - a counseling service using emotional response generation", Dongkeon Lee ; Kyo-Joong Oh ; Ho-Jin Choi, 2017 IEEE International Conference on Big Data and Smart Computing (BigComp)

Early study tries to use chatbot for counseling services. They changed drinking habit of who being consulted by leading them via intervene chatbot. However, the application did not concerned about psychiatric status through continuous conversation with user monitoring. Furthermore, they had no ethical judgment method that about the intervention of the chatbot. We argue that more reasonable and continuous emotion recognition will make better mental healthcare experiment. It will be more proper clinical psychiatric consolation in ethical view as well. This paper suggests a introduce a novel chatbot system for psychiatric counseling service. methods with emotion recognition. It senses emotional flow through the continuous observation of conversation. Also, we generate personalized counseling response from user input, to do this, we use additional constrains to generation model for the proper response generation which can detect conversational context, user emotion and expected reaction.

\section{PROPOSE SYSTEM}

In Proposed system we are developing a chatbot application using NLP..Its is a web application in which - User can ask a question on that chatbot. When user can ask a question then it can upload a file on that database. So admin can match those file and give a answer to that question. Admin is also responsible for managing module.

\section{SYSTEM ARCHITECTURE}

Following diagram is our system's architecture diagram:

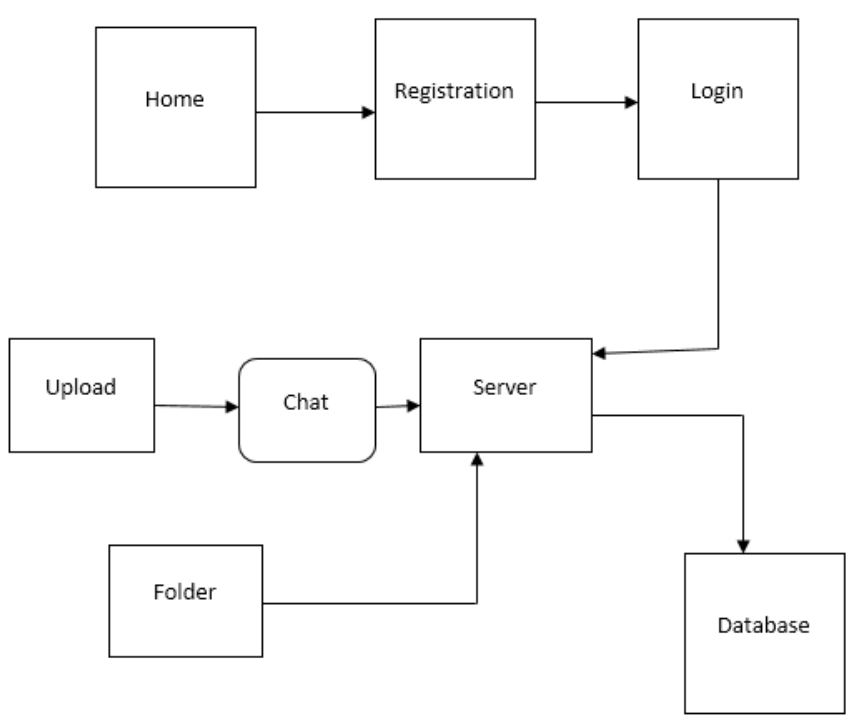

Figure 1: system architecture 
Vol. 8, Issue 5, May 2019

In system architecture User get register with the application with unique id and password. For login purpose user use same id amd password, after successfully registration the user get authority to acess system.

In next step after successful registration process, user upload file in NLP format. Uploaded file get trained by system and get save in the database use in this application.After completion of all this process, user can ask question to chatbot system that he/she want and get answer of it accordingly.

\section{METHODOLOGIES}

Chatbots are the programs that are designed to simulate conversations with humans and respond to different input modulities such as natural language text in human like manner. Its advantage is the possibility of performing repetitive tasks given specific commands or requests while it gives the impression that it can understand and give answers send message.

\section{Natural Language Process:}

\section{Algorithm Inputs}

1. Input question:-User input/ask the question based on uploaded file to the system.

2. Streaming question:-The question related to the uploaded file and user input get display in queue.

3. Selecting Object:-The system select the object from user question and match with streaming question

4. Read file content and splitting line by line:-The system read file content and split the user ask question line by line to match with streaming question

4. Check Object line by line:-A system check input question /object and generate the answer base on ask question, uploaded file using streaming question and other data.

5. Append Answer:-Generated answer get append with question anad get display to the user

6. Return answer:-User get display ask question along with generated answer through system.

All the ask question, streaming question generated answer and other data get stored in MYSQL database.

\section{ADVANTAGES}

1. Accesible Anytime:- Chatbots are basically virtual robots they never get tired and continue to execute your command. They will continue to operate every day throughout the year without requiring to take a break.

2. Flexible attribute: Chatbots have the benefit that it can quite easily be used in any industry.

3. Communication with user is easy.

4. Reduces Human Effort for reading files.

5. Easy for maintainance.

\subsection{Disadvantages}

1. More than one user can not be answer at a time.

2. Only specific format file should be given.

\section{APPLICATION}

- $\quad$ The proposed system can be used by farmers individually.

- It is can also be used in soil investigation centres.

\section{CONCLUSION}

The device proposed here is an interactive Application, which is capable of answering a questions and Answer. We propose to develop interactive educational software which can run on the desktop. The software helps the user to get answer without reading the file. Initially the software is given input with the file of specific format . Most of the working people in India by using this software will save time and get answer on click. In Future Scope Chat Bot application can be build for the various different fields in different domains along with various domain. For Ex, For communication for Medical, Collages and other important purpose . 


\section{RESULT}
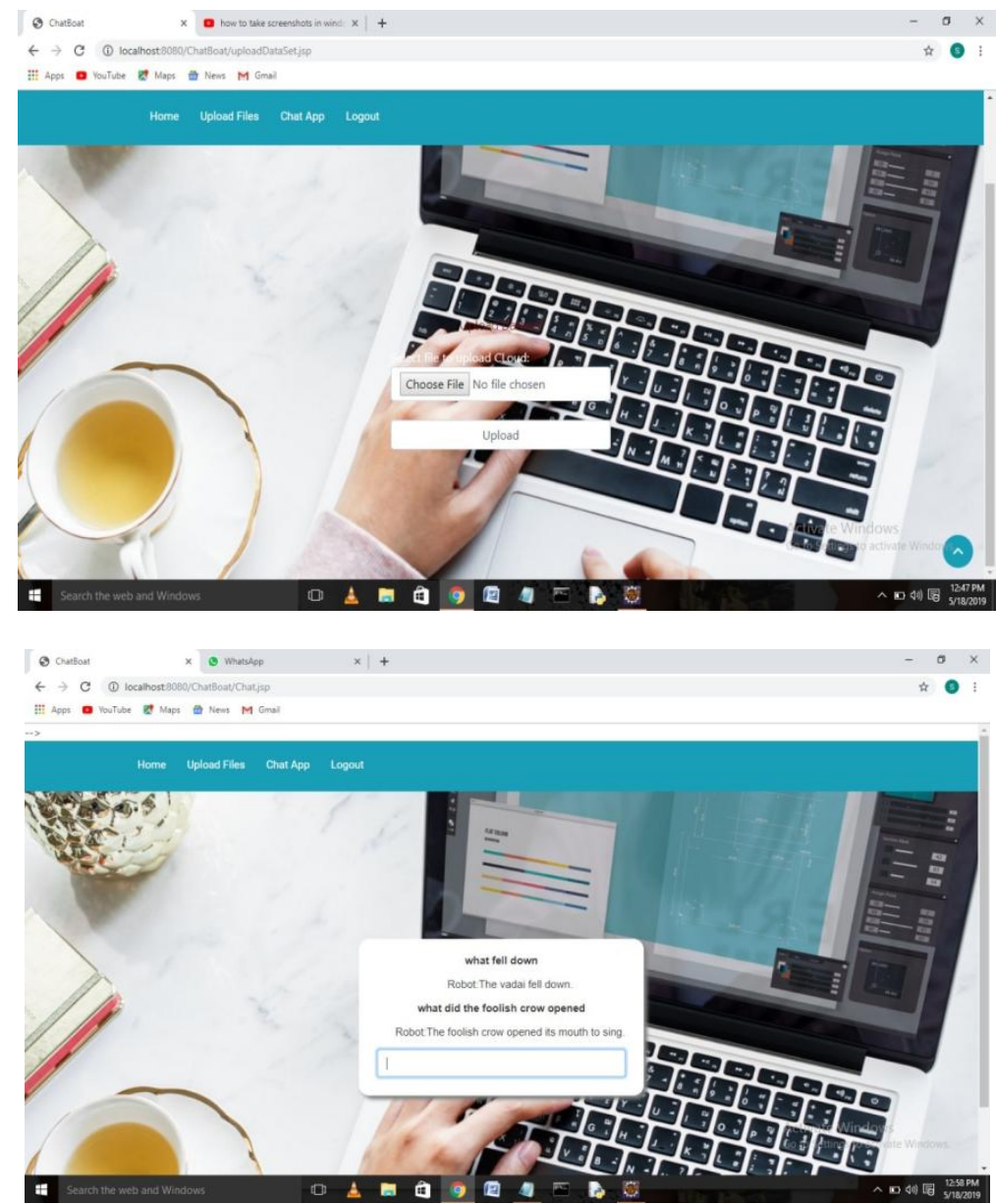

\section{REFERRENCES}

[1]. Lili, Z., amp;amp;amp; Yanli, W. (2010). Design of Harmonious HumanComputer Interaction Based on Intelligence Technology Development. 2010 IntRanoliya, B. R., Raghuwanshi, N., Singh, S. (2017). Chatbot for university related FAQs. 2017 International Conference on Advances in Computing, Communications and Informatics (ICACCI). doi:10.1109/icacci.2017.8126057ernational Conference on Intelligent

[2]. System Design and Engineering Application. doi:10.1109/isdea.2010.17

[3]. DHaro, L. F., amp;amp;amp; Banchs, R. E. (2017). Learning to predict the adequacy of answers in chat-oriented humanagent dialogs. TENCON 2017 - 2017 IEEE Region 10 Conference.

[4]. doi:10.1109/tencon.2017.8227907

[5]. Joseph Weizenbaum. ELIZA a computer program for the study of natural language com- munication between man and machine. Communications of the ACM, 9(1):36 45.

[6]. Richard S. Wallace. Be Your Own Botmaster: The Step By Step Guide to Creating, Hosting and Selling Your Own AI ChatBot On Pandorabots. ALICE AI foundations, Incorporated

[7]. Ranoliya, B. R., Raghuwanshi, N., amp;amp; Singh, S. (2017). Chatbot for university related FAQs. 2017 International Conference on Advances in Computing, Communications and Informatics (ICACCI). doi:10.1109/icacci.2017.8126057

[8]. Ly Pichponreay, Jin-Hyuk Kim, Chi-Hwan Choi, Kyung-Hee Lee, amp;amp; Wan-Sup Cho. (2016). Smart answering Chatbot based on OCR and Overgenerating Transformations and Ranking. 2016 Eighth International Conference on Ubiquitous and Future Networks (ICUFN). doi:10.1109/icufn.2016.7536948

[9]. Thomas, N. T. (2016). An e-business chatbot using AIML and LSA. 2016 International Conference on Advances in Computing, Communications and Informatics (ICACCI). doi:10.1109/icacci.2016.7732476

[10]. Bhawiyuga, A., Fauzi, M. A., Pramukantoro, E. S., amp;amp; Yahya, W. (2017). Design of E- commerce chat robot for automatically answering customer question. 2017 International Conference on Sustainable Information Engineering and Technology (SIET). doi:10.1109/siet.2017.8304128

[11]. Niranjan, M., Saipreethy, M. S., amp;amp; Kumar, T. G. (2012). An intelligent question answering conversational agent using Nave Bayesian classifier. 2012 IEEE International Conference on Technology Enhanced Education (ICTEE). doi:10.1109/ictee.2012.6208614

[12]. Shah, A., Jain, B., Agrawal, B., Jain, S., amp;amp; Shim, S. (2018).

[13]. Problem solving chatbot for data structures. 2018 IEEE 8 th Annual Computing and Communication Workshop and Conference (CCWC). doi:10.1109/ccwc.2018.8301734

[14]. Satu, M. S., Parvez, M. H., amp;amp; Shamim-Al-Mamun. (2015). Review of integrated applications with AIML based chatbot. 2015 International Conference on Computer and Information Engineering (ICCIE). 